\title{
\begin{tabular}{l|l} 
Mibraries & DSpace@MIT
\end{tabular}
}

\author{
MIT Open Access Articles
}

\section{Build Your Firm With Strangers?: Longitudinal Studies on Open Source Hardware Firm Growth}

The MIT Faculty has made this article openly available. Please share how this access benefits you. Your story matters.

Citation: Li, Zhuoxuan and Warren Seering. "Build Your Firm With Strangers?: Longitudinal Studies on Open Source Hardware Firm Growth." Proceedings of the ASME Design Engineering Technical Conference, August 2019, Anaheim, California, USA, American Society of Mechanical Engineers, November 2019. (C) 2019 ASME

As Published: http://dx.doi.org/10.1115/detc2019-97618

Publisher: American Society of Mechanical Engineers

Persistent URL: https://hdl.handle.net/1721.1/126834

Version: Final published version: final published article, as it appeared in a journal, conference proceedings, or other formally published context

Terms of Use: Article is made available in accordance with the publisher's policy and may be subject to US copyright law. Please refer to the publisher's site for terms of use. 


\title{
BUILD YOUR FIRM WITH STRANGERS?: LONGITUDINAL STUDIES ON OPEN SOURCE HARDWARE FIRM GROWTH
}

\author{
Zhuoxuan Li ${ }^{1}$, Warren Seering ${ }^{2}$ \\ Massachusetts Institute of Technology \\ Cambridge, MA
}

\begin{abstract}
The success of many open source software projects revealed the power of voluntary collaborative production of large/complex software systems. The research community is therefore curious about the viability of open source projects in other areas. In around 2000, open source practices started to take place in the commercial hardware realm, and so far, the phenomenon has not been fully explored. Using grounded theory, the authors studied 31 firms for an average of 2.3 years, discovering a 4-phase growth pattern of open source hardware firms, including starting the firm, identifying core competencies, business model improvement and business maturation. The firms' behaviors in each stage are reported, as well as the evolution of community demography, behaviors and impact in different growth phases.
\end{abstract}

Keywords: open source hardware, entrepreneurship, core competence, and business model sustainability.

\section{INTRODUCTION}

Viewing the success of open source software projects, researchers have asked whether open source makes sense in the hardware realm. In fact, in the past 15 years, the inflow of open source spirit did create a few very impactful hardware projects such as RepRap ${ }^{2}$ and Arduino ${ }^{3}$, which fundamentally changed how people design and make.

In general, open source takes place in the following way in the hardware realm: a project initiator releases the source design files of a hardware product on the internet, drawing attentions of interested people and so forming the initial community of the project. The community then voluntarily co-designs or improves the product without asking for monetary reward $(\mathrm{Li}$, Seering

\footnotetext{
1 Contact author: zxli@mit.edu

2 https://reprap.org, open source self-replicable 3D printer project

3 https://www.arduino.cc/, open source single board microprocessor project
}

2019). This phenomenon has already been thoroughly studied in the area of Free/Open Source Software (von Krogh et al. 2012). However, due to its physical nature, a hardware product cannot really be co-produced as its software analogs whose design and production naturally take place simultaneously. In reality, the solely online sharable part of a hardware product is its design. The utility of the open source hardware design is never free as with software but requires one to at least spend time and money to turn the design into an actual object. Therefore, the potential of open source in the hardware realm becomes an intriguing but complex research topic.

There are many approaches to explore open source hardware. The area we are particularly interested in is its economic potential. Over 4 years, the authors have observed that instead of filing a patent, many entrepreneurs opened the design of their core products to start their entrepreneurial journey, which is quite against the traditional business school style of entrepreneurship. The authors wish to understand whether this open source behavior is a new paradigm of conducting entrepreneurship or just a bold attempt by inexperienced, irrational entrepreneurs. This paper follows up our previous research (Li et al, ICED 2019), in which we explained the paradox of using an open source strategy in new firms. Briefly, open source brings community value inwards allowing new firms to create higher customer perceived value and to lower their development, recruiting, customer acquisition and managerial cost. However, open source also lowers the marketentry barrier for competitors by saving the effort to re-invent the product and to prepare for patent infringement.

After understanding the paradox, we then asked, "How do new open source hardware firms each build their sustainable 
business model over time?" Studying 31 open source hardware firms, we discovered a temporal 4-phase growth pattern; Starting Firm, Identifying Core Competency, Business Model Improvement, and Business Maturation. We further explained the community's impact on different phases in hopes of inviting other researchers to compare the open source model with the traditional closed source entrepreneurship model.

Our research outcomes contribute to the research body of "open design", "design and entrepreneurship", "open innovation" and "free innovation". They also bridge the gap between design research and management research regarding the impact of open practices in new firms. This paper addresses the potential interests of entrepreneurs who have already opened their hardware products; pre-entrepreneurs who opened their hardware projects for non-economical purposes but now want to start firms; entrepreneurs or entrepreneurial managers who are interested in using open source strategies; venture capitalists who are looking for metrics to measure the open source firm's value; policy makers who care about the social welfare of technologies; and educators of design, management, innovation, and entrepreneurship.

\section{DEFINITIONS AND LITERATURE REVIEW}

\subsection{Definition of an open source hardware firm}

The definition of open source hardware, according to the Open Source Hardware Association" is "Open source hardware is hardware whose design is made publicly available so that anyone can study, modify, distribute, make, and sell the design or hardware based on that design."

To be considered as an exemplar in this paper, a firm needs to meet three criteria. First, the firm must have at least one open source hardware product whose product blueprints, CAD files, software code, and assembly instructions are all available online and licensed under an open source license within two years of firm's incorporation. The design files need to demonstrate transparency, accessibility and affordability (Fjeldsted et al. 2012). Second, the firm must demonstrate an active community relationship, i.e., on average more than 1 post/per month on the firm's online forum. Third, the firm must have already generated or have actively attempted to generate revenue via the open source product.

\subsection{Literature review}

Research studies on the commercialization potential of open source hardware products have two major branches. One branch is about exploration of a potential revenue model. Based on the observed revenue models of open source software, researchers have built some conceptual revenue models about open source products. Salem and Khatib analyzed the revenue streams of open designed electronics (Salem \& Khatib 2004); Pierce stated the market potential of open source scientific equipment in the scientific and maker community (Pearce 2017). Howard

4 https://www.oshwa.org/ suggested how project initiators could make revenue from open designed products (Howard et al. 2012). A second branch addresses use of existing business model tools to describe the activities in commercial open source hardware products. Fjeldsted et al proposed platform and community to be new components in a business model canvas (Osterwalder 2015) for open source firms (Fjeldsted et al. 2012). Moritz et al used a business model canvas to study Open Source Ecology ${ }^{5}$ (Moritz et al. 2016). Bonvoisin et al focused on the relationships between different components in the canvas and studied 23 both nonprofit and for-profit open source projects to report an observation about relationships between the company, the customer and competitors (Bonvoisin et al. 2017). So far, most research outcomes are conceptual and thus not intuitive to apply in practice. Additionally, most studies haven't distinguished economical purposes of the focal projects, which might result in inaccurate interpretation of observed behaviors. Moreover, many research papers have taken a holistic and static view of the life cycle of an open source hardware product, though it is obvious that a more mature open source project has more likelihood to have more resources.

Open source related practices were also studied in other disciplines. For example, Open Innovation theories, in organization management research, appeal to firms to open their IP and take advantage of external resources (Chesbrough \& Appleyard 2007). User Innovation theories, in innovation research, advocate that firms should pay attention to customer innovation and co-innovate with users (Hippel 2007).

\subsection{Taxonomy of the community's role}

The community is a stakeholder in the evolution of open source hardware firms. The definition of community is grounded from our interviews with the founders of open source hardware firms who believe their communities to be "people who are not employed by the firm but have contributed to the firm's sales, marketing, technology or business development." When a firm makes its product's source files open, it naturally attracts techenthusiasts to explore the design of products, forming the snowball seed of the company's community.

The community impacts the firm through direct or indirect, online and offline interactions. In our previous research, by studying 5 open source hardware firms' online forums, we grouped different community - firm interactions into five major categories - development, business resources, product Q\&A, community facilitation and help seeking (Li et al. ICED 2019) (see Table 1).

\section{TABLE 1: COMMUNITY IMPACTS AND ROLES}

\begin{tabular}{l}
\hline Community Roles (exemplified in the case of a humanoid robot) \\
\hline Development \\
- Core developer: shares, co-designs or refines the core structure or \\
components of the open source hardware product. Mostly joins the \\
community in the very beginning E.g., a core developer helps \\
\hline
\end{tabular}

${ }^{5}$ https://www.opensourceecology.org/, a non-profit open source project where modular industrial farming machines are co-designed by the project's internal team and its community 
developing the servo system (controlling the movement of legs and hands) for a humanoid walking robot.

- Feature developer: develops new or refines existing features related to the product. Mostly are buyers and join the community after starting sales. E.g., a function developer develops the vocal system by adding a mini speaker hidden inside of the robot body.

-App developer: develops new or refines existing applications. Mostly join in the community after products have APIs or become programmable. E.g., an app developer develops a new dance for the robot using the robot's graphic programming interface.

\section{Managerial activities or resources}

-Mentor: helps firm planning business strategy, joining during the early stage.

-Resource introducer: introduces financial, HR, manufacturing, partnership resources to the firm, joining during the early stage

-Information collector: shares market news, competitor info or other opportunity information.

-Advertiser: writes blogs, sharing news about the firm in their personal networks

\section{Product Q\&A}

-Active consumer: gives feedback on user experience, express needs, and propose new ideas, features or recommendations to the firm.

- Normal consumer: reports issues about product utilization and seeks solutions

\section{Community facilitation}

- Tech facilitator: answers technical questions.

- Non-tech facilitator: proposes or organizes online or offline meetings, giving suggestions for documentation, forum management etc.

\section{Help seeking}

Help-seeker: seeking for expertise in the community E.g., a master student working on robotics is seeking help from the community about the robot balancing issue.

\section{RESEARCH METHODS}

The research question in this paper is to understand "How new open source hardware firms each build their sustainable business model over time." It falls into the exploratory research realm (Yin 2016). The phenomena of entrepreneurs running an open source hardware firm only emerged in around 2000, and the total volume of open source hardware firms worldwide is still very small. Therefore, we choose grounded theory as our major research method (Charmaz 2014). Grounded theory is suitable for uncovering hidden theories or building new theories about novel social phenomenon. In practice, researchers immerse immediately into the social activities without preloading any existing theories in mind. Using observation, interview, and survey to collect qualitative data, researchers can then code the field memos and interview transcripts to generate codes. For example, when we are exploring the core competitive competences, we asked the CEO of M5Stack “... What did you do to increase sales? Why did you believe it could work at that time?" He answered, "I think customers are not stupid, they can distinguish good qualities or bad qualities. We are only doing good quality stuff with a very low price." Therefore, we coded "quality", "low cost". Next, we had two trained research assistants group the codes into different categories and we compared, discussed and agreed on the grouping results. We then developed the categories into a theory which we compared to existing theories to determine whether it is just an alternative statement of some exiting theory (Charmaz 2014). We picked 31 cases for longitudinal research and interviewed each founder three or more times to get a detailed history about the firms' growth.

Over four years, we have accumulated a base of about 127 global open source hardware firms from 23 countries - China, Japan, Singapore, Russia, India, the USA, Canada, Brazil, Australia, New Zealand, Germany, Italy, France, Switzerland, the UK, Ireland, the Netherlands, Denmark, Finland, Norway, Estonia, the Czech Republic and Spain. The data collection started with Wevolver ${ }^{6}$ an online open project collaboration platform, to get the initial 21 firms interviewed. Then, we were introduced to other open source hardware firms by these 21 firms. We also collected our sample by going to Maker Fairs in New York City and Shenzhen from 2015 2017.

The first step of data collection was to identify the historical activities of the targeted firms. We collected historical data in three ways. The first way was to acquire the companies' online archival data from scraping the firms' blogs, newsletters, forums, Facebook or Twitter groups, as well as media reports. Using Wayback Machine ${ }^{7}$, we also managed to track the firms' website content changes over time. The advantage of using archival data is that we are able to get a quick and holistic understanding of the firms' growth independent of availability of the firm's founders. The flaw is that the information is often too fragmented to form a consistent firm growth picture, and we also cannot get the reasons why a firm evolved the way it did.

We then developed our interview strategy. Questions for the first round interview were designed in a semi-structured way with open questions such as "Tell me the story about your company?" or "Why did you open source your product?", "What are some milestones in the firm development?", "Why do you think they are milestones?" When founders mentioned information related to what we found from archival data, we confirmed our observations with them to assure the data accuracy. After the first round of interviews with 13 companies in 2 months (January and February 2016), a pattern of 4 phases of open source hardware firm development started to show up. In 2016 and 2017 July and August, we launched our second and third round interviews with first round firms and newly collected firms to test the validity of our observed pattern and to understand more deeply about the four stages. We added specific questions, such as "When did you start to feel you wanted to commit to the firm?" "Community, product, organization, which order do you think your company had? (Interviewee was told the meaning of community, product, and organization before answering)." After these 23 cases, we didn't see new features appearing in this pattern. We continued to study another 8 companies until we felt the sample size was enough to support a thoughtful analysis.

\footnotetext{
6 https://www.wevolver.com/
} 


\section{FINDINGS ABOUT FIRM GROWTH}

\subsection{Phase I: Starting the Firm \\ 4.1.1 The firm's birth}

The start of most open source hardware firms is very often unplanned and disorganized and may even lack commitment from the founder(s). Very few founders started their firm from a well-prepared "business plan"; instead most merely treated the product as a hobby project, and their incentives for open source actions come from morality or social responsibility or simply to get more "playmates". If the design proved to be useful or playful for community members, the community might adopt the open design and customize it according to their needs. If the founder or someone else in the community demonstrates enough effective engagement with other community members, he automatically becomes the leader of the community. Later, because of the community's word of mouth, more and more people might be drawn to the project and some would express their willingness to buy parts, kits or assembled products, pushing the leader to think about quitting his job and turning the project into a business. Thus, the birth of an open source hardware firm is always entangled with validation of the product's design with help from the community, as well as the founder's self-realization as a full-time entrepreneur.

We identified three major events for our sample firmsvalidation of product design $(\mathrm{P})$, formal incorporation(I), and formation of community (CM). Validation of design confirms technological feasibility of the product. Formation of a community reflects market interests. There are 6 temporal patterns that the three events can form. In Table 2, we list firms' birth patterns exemplified by one interviewed firm for each. Figure 1 shows the distribution of patterns. Interestingly, about $50 \%$ of firms did not start with a formal incorporation.

\section{TABLE 2: SIX BIRTH PATTERNS AND EXAMPLES}

\begin{tabular}{l}
$\mathbf{P}-\mathbf{I}-\mathbf{C M}$ \\
Ufactory ${ }^{8}$ is a Chinese robotic arm design and manufacturing firm \\
whose founder used his Master thesis research project (P), an open \\
source robotic arm made of laser-cut pieces, to start the firm. He \\
decided to open source his design after the incorporation (I) in hopes \\
of attracting customers. For the first year he didn't intentionally \\
engage with his community. A steady online community formed after \\
he started community management (CM). \\
\hline $\mathbf{P}-\mathbf{C M}-\mathbf{I}$ \\
OpenMV ${ }^{9}$ is located in Texas, USA. It makes a programmable \\
camera lens module. The product was originally invented by an \\
Egyptian engineer (P) and open-sourced on a maker platform, \\
Hackaday.io. The platform members then started to discuss, replicate \\
and improve the product. The project was so popular in the \\
community that it was selected as the best project of the year (CM). \\
An American engineer showed interested in commercializing the \\
module, so in 2016 OpenMV was founded as a for-profit startup (I).
\end{tabular}

\footnotetext{
https://www.ufactory.cc/\#/

9 https://openmv.io/

10 https://www.openrov.com/

11 https://3dr.com/
}

$\mathbf{C M}-\mathbf{P}-\mathbf{I}$

Located in California, OpenROV ${ }^{10}$ is a spinoff firm from an underwater exploration amateur community (CM). The two cofounders initiated the group to meet other underwater exploration amateurs. The community co-designed the first prototype of OpenROV (P). Later, more and more people wanted to purchase the prototype, so the two co-founders incorporated their company (I).

$\mathbf{C M}-\mathbf{I}-\mathbf{P}$

3DRobotics ${ }^{11}$ is a drone service company located in California, USA. The founder started a UAV community - DIYDrone (CM), where hobbyist can share and co-work on the UAV designs. The founder found the business opportunity to sell low-cost UAV hardware and software, so he started his company 3DRobotics(I) and developed both hardware products and software services $(\mathrm{P})$. $\mathbf{I}-\mathbf{C M}-\mathbf{P}$

Sparkfun ${ }^{12}$ started as an electronic component retailer in Colorado, USA (I). Before designing and selling their own open source electronics, they had already accumulated a community from the website forum where buyers discussed and shared issues and tricks (CM). It finally turned into an open source electronic design and manufacturing company $(\mathrm{P})$.

\section{$\mathbf{I}-\mathbf{P}-\mathbf{C M}$}

Dobot $^{13}$ is a Chinese robotic arm design and manufacturing company for beginner-level robotics learning. It incorporated in 2013 and products were developed in house (I). Seeing the wave of open source, the owners decided to open the design of their robotic arm and provided a lot of free online learning materials $(\mathrm{P})$, making their reputation in the maker community $(\mathrm{CM})$.

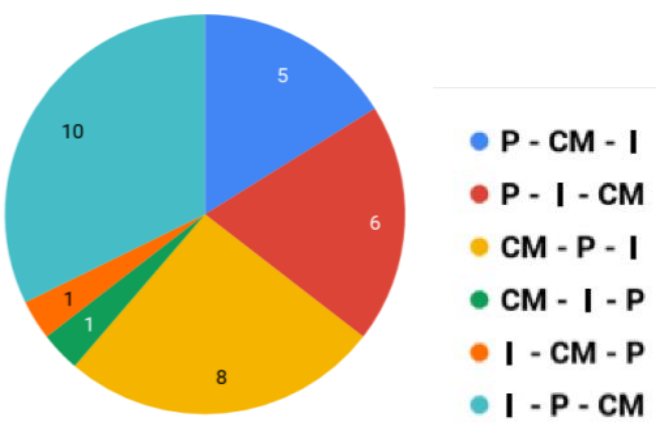

FIGURE 1: SIX PATTERNS OF FIRM BIRTH

\subsubsection{First Revenue Model}

Without patents, open source entrepreneurs are normally not in the favor of traditional venture capital managers (Haeussler et al. 2009). Lack of financial resources becomes the first barrier to the entrepreneurs in starting their business. Additionally, though an open source firm can have a supportive community and a community-validated design even before it has sales, it is still unclear whether the mass market would be willing to pay when the design is freely downloadable. A business viability willingness to pay is crucial to keep open source entrepreneurs confident in continuing their journey.

\footnotetext{
12 https://sparkfun.com/

13 https://www.dobot.cc/
} 
We observed that apart from selling the product directly on the firm's home website, most open source firms have chosen crowdfunding in order to test market attitudes towards the product concept (Palacios et al. 2016). Once it turns out that people support the firm's crowdfunding campaign, the entrepreneur would be more convinced about the product's market potential, and under less financial pressure in production. Among our sample firms, we have 17 out of 31 companies which reported to have launched crowdfunding campaigns for their open source product (See Figure 2 (a)). Zack, the community manager and third employee of OpenROV, explained to us about their first crowdfunding campaign "...Of course we were worried, but they (the community) backed us immediately and promoted us on other websites during our Kickstarter campaign. That's why we only used THREE days to achieve our goal... They gave us confidence to continue..."

\subsubsection{Recruiting from the community}

The community also serves as a talent pool for the firms' recruiting. New firm recruiting has been well recognized as a key issue for firms' survival, as the opportunity cost of a wrong hiring is so high that it can be fatal to the firm. 24 out of 31 firms reported that they recruited at least one employee in their first 5 employees from their early community (See Figure 2 (b)). Interestingly, the firms who reported to have hired from their virtual community have all spent at least 5 hours per week (1 hour per day) to engage with the virtual community.

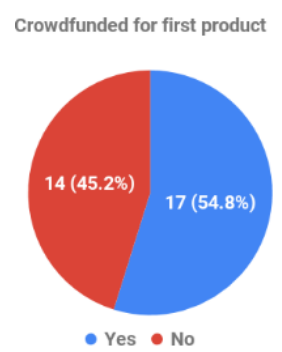

(a)

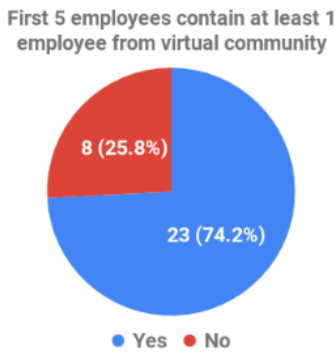

(b)

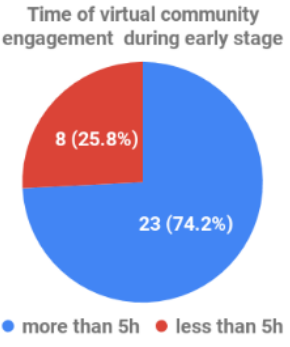

(c)
FIGURE 2: STATISTICS OF CROWDFUNDING, RECRUITING AND COMMUNITY ENGAGEMENT

\subsection{Phase II: Identifying Core Competencies}

After validating technology feasibility and business viability, an open source hardware firm comes to the phase of identifying and building its core competencies. Theoretically, if a firm could forever stay a monopoly, even in a niche market, it could comfortably charge a profit maximizing price and generate more and more profit over time as long as the market is growing. However, in reality, when an open source firm proves business viability, competitors may also decide to enter the market with a shortcut of free design files. Core competencies are areas of deep proficiency that can help distinguish a company from its competitors because they are hard for competitors to copy or procure (Bain\&Co.). Understanding core competencies allows

14 https://www.adafruit.com/ companies to invest in the strengths that differentiate them and set strategies that unify their entire organization. Though 31 cases, we identified five core competences - Community Excellence, Quality/Price Excellence, Fast Innovation Capability, Community-Networking Effect, and Ecosystem. See Figure 3.

\subsubsection{Community Excellence}

Community Excellence means the firm differentiates itself by building an inclusive community culture to incubate an active community. Revenue can then be generated in a sustainable way since 1) the community performs recurrent purchasing behaviors to try new things because they are frequently impressed or inspired by shared creative projects; 2) the community has a strong word-of-mouth so the firm is able to acquire new customers with low cost; 3 ) the firm manages to quickly convert new customers into the firm's loyal community members.

Adafruit ${ }^{14}$ possesses a core competency of community excellence. Adafruit is an electronic components manufacturer and retailer located in New York City. The firm prioritizes issues related to community culture building and they call themselves a community-driven firm instead of a product-driven firm. As Adafruit's primary targeted customers are the beginner-level makers, the firm designs their community culture as inclusive and learning-encouraging. Their online store is like a free online course platform for beginner-level makers. Most products are provided with detailed, comprehensive tutorials. The tutorials are videoed by the CEO and founder, Ms. Limor, in person and are updated frequently. She treats every viewer as a beginner, telling experienced viewers which parts to skip. It turns out this action shortens the distance between firm and its community, as we can see from the comments under each video. Adafruit makes new product plans according to their community's data. Adafruit also promises the fastest delivery, free return or exchange and professional tech support to their community. The firm has frequent open office days, workshops, competitions and celebrations to connect with their community offline. Ms. Limor told us that most community members could feel the efforts and care from the firm, and they are happy to help building a more connected, inclusive culture by sharing their projects or by helping other community members to succeed.

Community excellence as a core competency is more likely to appear from an early phase of firm growth. Open source hardware firms who choose community excellence as a core competency focused on minimizing the cost of the community's learning, communication and sharing activities.

\subsubsection{Quality/Price Excellence}

Quality/Price Excellence means that the firm focuses on controlling product quality and cost to outperform its competitors in order to generate sustainable profit. Lulzbot ${ }^{15}$ is a spin-off firm from RepRap that is building open source 3D printers in Colorado, USA. After the price war in the DIY 3D printer market, Lulzbot founder, Jeff Moe, realized that to successfully compete against cheaper Chinese manufacturers, they needed to focus on the quality of pre-assembled 3D printers

15 https://www.lulzbot.com/ 
and to minimize the cost. Lulzbot built its own manufacturing lines in 2014 to control the quality and cost of their products. It also uses lean manufacturing methods to manage the production cost.

Unlike Lulzbot which keeps all its products open source, many firms, whose core competency is also quality/price excellence, closed their new products, such as OpenROV, Dobot, Ufactory, and Microduino ${ }^{16}$. They reasoned that the potential buyers valued quality/price more than being open or hackable. The designs of their new products are more integrated with pieces injection-molded and preassembled to increase the quality and decrease price.

\subsubsection{Fast Innovation}

Fast innovation means the firm develops the capability of inventing novel products in a fast way. Normally, a new open source product targets a niche market and provides novel solutions. Unlike open source software products, copycat competitors in open source hardware, even with the design being free and accessible, always need time to figure out a costeffective method to manufacture, assemble and distribute. The economics behind fast innovation is that by innovating quickly, the open source firm can stay ahead of copycat competitors for a certain period of time and be a monopolist in the market. When copycat competitors enter the market competing on price, the open source firm can also lower their price and meanwhile start to sell new products as a monopolist in a new niche market. This phenomenon was also observed in the case of big firms, such as Intel and AMD (Calo 2011).

Sparkfun is an electronic component design, production and retail company. They target the medium-advanced level maker market, which is niche but not price sensitive. Sparkfun has a team of expert-level electronic engineers. The CEO himself is also a professional electronic engineer with deep insight in electronics and the maker industry. They have their community managers identifying ideas of new products from their community forum and passing the ideas to their internal exploratory research lab, XLab, to prototype several trial pieces. Then they distribute the trial pieces to their community members for free, launching workshops or competitions in order to test the demand for large volume production. The CEO of Sparkfun told us that their average new product go-to-market timespan was 12 weeks and they owned an internal manufacturing plant for new products manufacturing.

Another inspiring example is M5stack, a single board microprocessor firm located in Shenzhen providing integrated and stackable open source electronic products. Compared to Sparkfun, M5Stack's innovation is not from the community but from its own CEO and founder who has been in the electronics industry for 2 decades and has deep insight and vision about the future of the IoT market. M5Stack's fast innovation capability comes from the execution capability of M5Stack's technology team and the rich resources in the Shenzhen ecosystem. M5Stack

\footnotetext{
16 https://microduinoinc.com/

17 https://farm.bot/
}

also owns its manufacturing resources and has developed 16 extensions in only 1 year.

If an open source firm wants to develop fast innovation capability as its core competency, it needs to have a community who actively report their innovative proposals, feedback and practices. The firm needs to have at least one professional community manager to prioritize and bring the data into the firm. The firm also needs to have an expert-level internal development team to develop new products in a low-cost way. Additionally, from the cases of Sparkfun and M5Stack, a firm may also have access to reliable and low-cost manufacturing resources.

\subsubsection{Community-networking effect}

Community-networking effect means one additional user using the product will result in more value for existing users. Community-networking effect appears a lot as a core competency in education-related open source hardware firms. The firm's forum serves as a platform with two parties - sharers and learners. If there are more sharers, there will be more learners. The community-networking effect comes into being when learning and sharing cost in the community is very low for a new user, and sharing behavior is appreciated and rewarded by the community. Thus, it is likely that after learning enough, a new user would become a sharer sharing his uses of the product and thus increasing the product value for existing users. This reinforces community growth. Community-networking effect allows the firms' products to become a standard of the market and the firm's community to become large and hence influential.

The Arduino project was originally designed by a group of Italian teachers for the purpose of teaching college students about electronics. The Arduino microprocessor itself cannot be used as an end product without writing code and attaching to hardware. If a user shares his projects using an Arduino board in the community, existing community members would have more options to use their boards by simply replicating the shared projects. Therefore, a new shared project increases the use value of Arduino boards for existing users. To achieve communitynetworking effect and to minimize the cost of learning and sharing, Arduino developed a very beginner-friendly coding tool, Arduino IDE, as well as a wiring framework, I/O system. These systems became the standards of the DIY microprocessor industry and universal tools for learning about electronics. The Arduino internal team also creates many easy-to-learn tutorials for beginners. To increase the quality of shared projects, Arduino is in strategic partnership with online project documentation platform Hackster.io to provide a better experience in sharing. To reward the sharers, Arduino has a community credit system to send free electronics or invitations to their events to sharers. Now, Arduino has become the biggest electronics community with a huge daily site visit rate and has become the first destination of electronics beginners.

Community-networking effect also serves non-education products. OpenROV and FarmBot ${ }^{17}$ are examples. OpenROV provides the global citizen underwater exploration platform ${ }^{18}$

\footnotetext{
18 https://openexplorer.nationalgeographic.com/home
} 
via which underwater amateurs are able to connect with each other and do missions together. The open explorer platform keeps attracting new customers to OpenROV and providing more expenditures from existing customers. Therefore, OpenROV's community has become the biggest underwater exploration community. FarmBot is an open source automatic farming system using $\mathrm{CNC}$ technology. The community platform encourages its members to learn farming skills for different plants from each other; they can also compete with each other on farming output. The community of FarmBot is now the biggest urban farming community.

To achieve community-networking effect, community size needs to achieve a critical level which triggers the follow-up exponential growth. It requires company leaders to identify the mechanism of community growth and design all the company's online or offline activities to promote the networking effect.

\subsubsection{Ecosystem}

Ecosystem as a core competency means an open source hardware firm connects its community, customers, suppliers, and partners in a value chain, distributing risks to all stakeholders, so that the open source firm becomes more robust in the presence of market change. Ecosystem as a core competency is more often observed in mature open source hardware firms. Being open source allows more stakeholders to design their business activities around the product. Using ecosystem as a core competency, company leaders need to design their business model in a more systematic way, linking different resources to make positive value loops. One risk is that inter-organizational management may become very complicated and costly in order to optimize each stakeholder's interests. An example is SeeedStudio $^{19}$ which sells open source products, runs the online marketplace for third parties to sell their open source products, provides design services and maker spaces, provides all-in-one idea-to-manufacturing services, and invests in customer projects to get returns. SeeedStudio has a sophisticated network of revenue resources from idea to manufacturing to market operations. Though, they have experienced accelerating sales from year $2005 \sim 2015$, in recent years they have started to confront the cost control issue due to too many stakeholders.

\subsubsection{Discussion of firms' core competencies}

Fast innovation and quality/price excellence as core competencies require open source hardware firms to have a capable community manager with an engineering-background to identify and prioritize the community's needs and to link the communnity's contribtutions to internal development. It also needs a capable internal technology team to develop profitmaximizing products using limited resources. Community excellence and community-networking effect as core competencies demand that firms put the community in the center of their business strategy. Ecosystem as a core competency needs the firm leaders to have a holistic view on the status-quo of the industry value chain. Firms also need to have access to abundant resources and strong bargaining power to make all parties cooperate in a low-cost way.

Product design and a business model can be copied, but not a firms' core compentencies. If an open source hardware entrepreneur deeply understands core competencies and purposefully invests in them, it is more likely that the entrepreneur will design and test business models in a quicker and more effective way. The core competences of an open source hardware firm depend on many factors, such as the experience and resources of the entrepreneur, the birth mode of the firm, the market competitive landscape and the characteristics of consumers. An open source hardware firm may possess one or multiple core competences at different strengthlevels (See Figure 3). A firm's core competences also can vary over the firm's maturation.

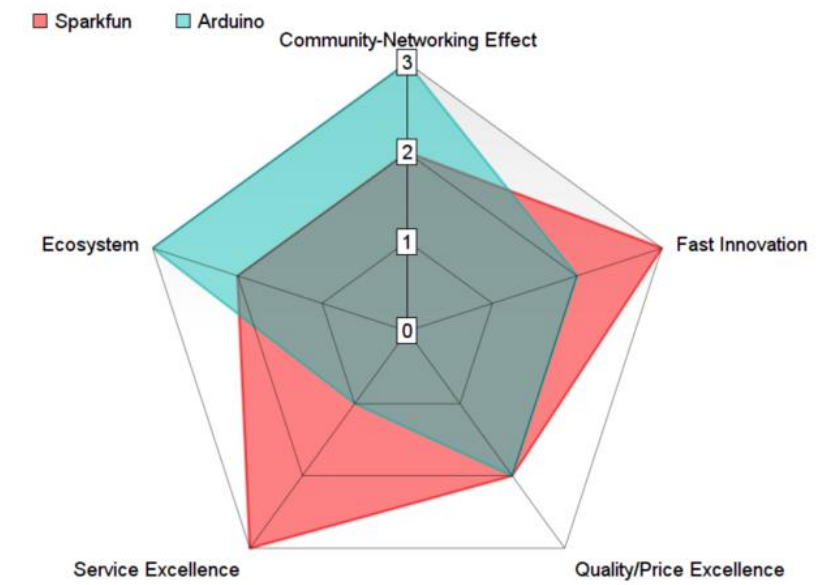

FIGURE 3: EXAMPLE OF COMPANY CORE COMPETENCE

\subsection{Phase III: Business Model Improvement}

Identifying core competencies and business model improvement tend to happen simultanously. Many interviewees reported that they identified their core competencies by trying different business models. If the tested business model works well, they may then allocate firm resources to strengthen core competencies in order to improve business model efficiency. Through interviews and achival data, we found that open source hardware firms generate profit from the following five revenue sources - Open Source Products, Closed Products, Community, Services, and Trademark Licenses (See Figure 4).

When a firm is making profit from selling open source products, they may sell unassembled kits, pre-assembled kits, replacement components and other accessories. Depending on the quality and cost of users' self assembling, some companies may decide to only sell the pre-assembled products, such as micoprocessor companies - Beagleboard ${ }^{20}$ and Lemaker ${ }^{21}$. On the contrary, some firms may decide only to sell kits and components to decrease the cost of assembling and delivery. An example is BlueRobotics ${ }^{22}$, an underwater robotics component company that also sells Solar powered underwater drone kits.

\footnotetext{
21 http://www.lemaker.org/

22 https://www.bluerobotics.com/
}

\footnotetext{
19 https://www.seeed.cc

20 https://beagleboard.org/
} 
When a firm is making profit from selling closed products, it may sell closed hardware or software products. For example, OpenROV sells both open source underwater drones as well as its equivalent closed version. According to the founder, the open source version is designed for hackers who want to tinker, while the closed version targets a broader mass market and ordinary customers who only buy to play. A firm may also profit from selling closed software products. Examples are 3DRobotics and Buzzbox ${ }^{23}$. An entrepreneur should take much caution when deciding to sell closed products since the community may feel betrayed, believing the firm is exploiting the community's contribution.

When a firm has a big enough community, it is able to gain profit from its community. The firm may charge third parties for advertising on the community forum. This model has a risk of losing the community because of a bad platform experience, such as too many banner ads. The firm can also introduce a third party to provide related products or services to its community, acting as a marketplace charging a fixed or commission fee from transactions. Examples are Sparkfun and Seeedstudio. Verifying the quality of market products is the key in this revenue model. Some open source hardware firms, like DronePlace, become organizers of competitions for community members, making profit from charging registration fees from participants.

The fourth revenue resource is selling related services. AI.frame ${ }^{24}$, a Shenzhen based open source robotics firm selling toy robots, provides large scale robot design and development services for theme parks or large events. Open Motors ${ }^{25}$, a California based open source modular automotive manufacturer, provides road security testing consulting services to automotive startups. Seeedstudio provides manufactuing support for small batch manufacturing. FaradayMotion ${ }^{26}$, a German open source electronic skate board firm, also provides local making classes in their own maker space.

Trademark licensing as a revenue resource is well exemplified by Arduino. The founder of Arduino reported that in 2013, the trademark license fee made up $10 \%$ of their total revenue.

\subsection{Phase IV: Business Maturation}

After building a firm's core competences and developing a sustainable business model to accumulate stable cash flow and adequate financial resources, firms enter the maturation phase. Open source hardware firms in this phase start to have a stable or increasing market share, profitable revenue models, a longterm business strategy, and resilience to competitions or market change. Open source firms in this phase either have a big market share and have made open source the norm for the market or have established a strong core competency and competitive advantages.

We illustrate the different phases and revenue resources of all interviewed open source firms in Figure 5. The boxed firms were no longer in the market by 2019 . The four phases are

\footnotetext{
23 https://www.osbeehives.com/

24 https://aiframe.com/
}

evolving in a continuous and smooth way. The characterization of a firm's phase and revenue resources were proposed by the authors and agreed to by the open source entrepreneurs.

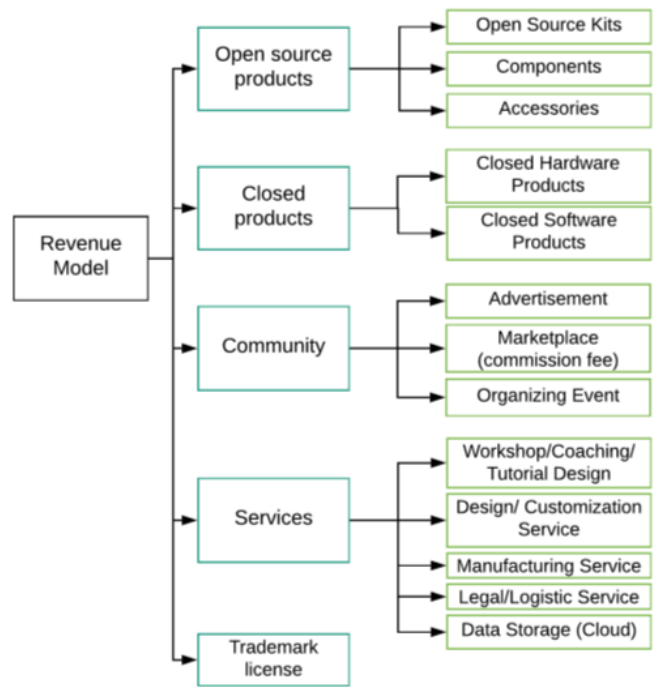

FIGURE 4: FIVE REVENUE RESOURCES

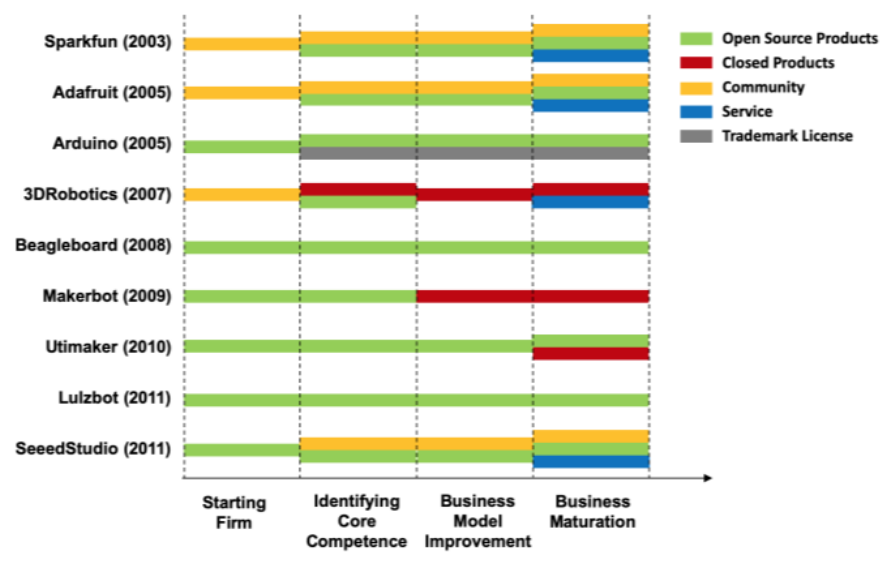

Figure 5 (a) - Firms who are in Phase IV in 2019

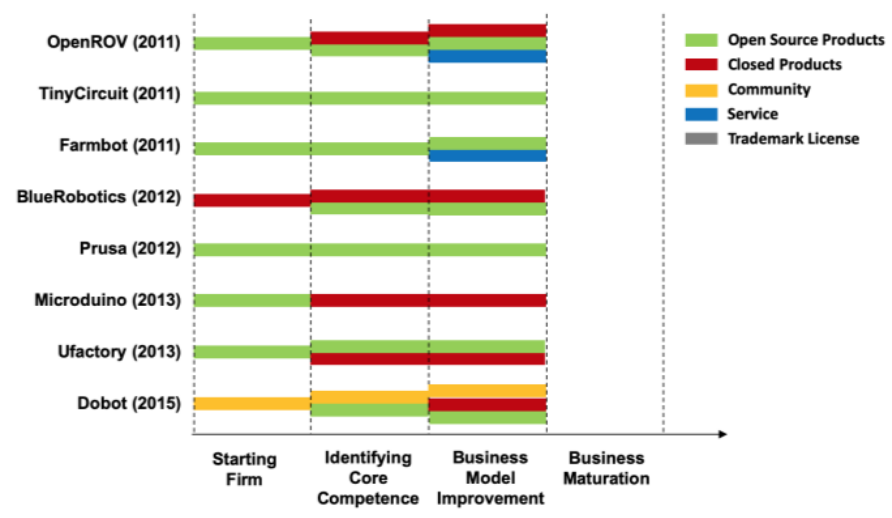

Figure 5 (b): Firms who are in Phase III in 2019
${ }_{26}^{25} \frac{\mathrm{https}: / / \text { www.openmotors.co/ }}{\mathrm{http}: / \text { www.faradaymotion.com/ }}$ 


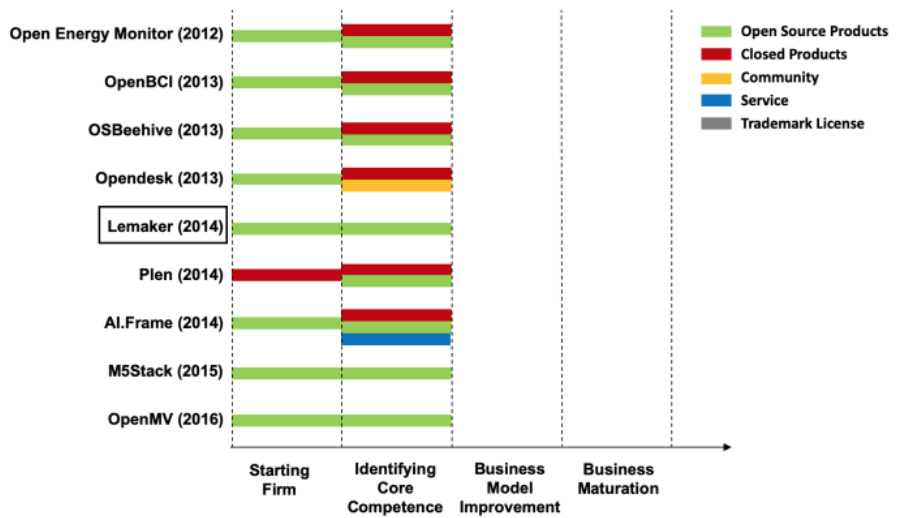

Figure 5 (c): Firms who are in Phase II in 2019

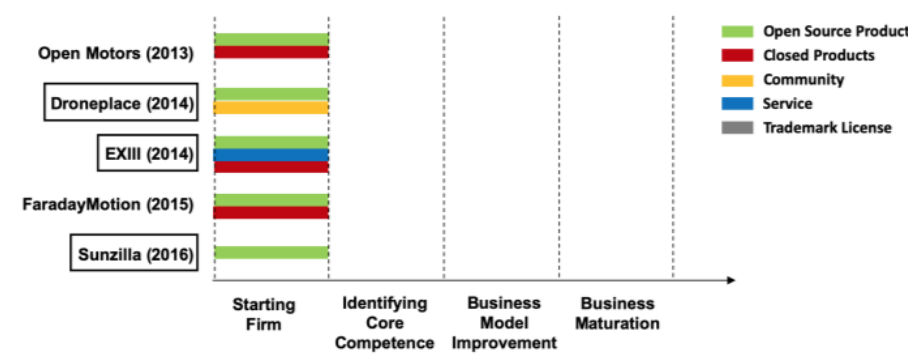

Figure 5 (d): Firms who are in Phase I in 2019

FIGURE 5: FIRMS' RENENUE RESOURCES IN EACH PHASES

\section{COMMUNITY IMPACTS ON FIRM DEVELOPMENT}

Through interviews we realized that the demography and behaviors of an open source hardware firm's community evolve together with the firm's development. According to interviewees, the community evolution, core competencies evolution and business model evolution dynamically entangle with each other. As the firm matures, the community's average technological expertise changes from tech experts to tech savvies and the community provides more marketing contributions than technical contributions.

In the phase of starting the firm, the community forms because of shared technological interests in the product's concepts or design elegance. The community possesses great potential in helping to improve the design by sharing its own projects to inspire internal teams or by testing the shared open design. In this stage, firms can use their community to get technological support to validate product technology and design. Interactions between the community and the firm are very active, which may result in a product's core design varying and iteration at a rapid pace in different directions. Therefore, we call the community in the starting phase "the developer community". Referring to Table 1, the majority of community members are core developers and tech-facilitators. Interestingly, we also observed the "Fans Effect" in the developer community. For example, in the M5stack community, one day its forum subscribers increased at an unexpectedly high rate, and community members started to discuss intensively about integrating the MicroPython Operation System into M5Stack. It turned out that a "Superstar" maker joined the M5Stack community and twitted the M5Stack product on his twitter. This attracted many of his followers to subscribe to M5stack, triggering the tide of operation system co-design between the firm and the community.

When the open source hardware product achieves its beta prototype level, firms may start to plan their first revenue model. The topics between firm and community may change to sales or marketing strategies, such as pricing, crowdfunding campaigns or sales channels. At this point we see mentors and resource introducers appearing in the community. They may first openly express their ideas and opinions on the forum. Then, they may prefer to chat with entrepreneurs privately to share experiences or help design the first sales campaign. The community now is like a "Mentor community".

In the phase of building core competencies, after firms successfully generate profit or get external investment, they may start to hire directly from their community to strengthen their core competencies. In this phase, the firm's community size typically grows rapidly with most new members as buyers instead of developers. The developer and mentor community members may become less active or even leave, since the internal team may not adopt their suggestions as often as before, or may not appeal to them to do as creative a job as before. Forking and spin-off (community members take the design and build their own firm) were observed during this phase. Meanwhile, founders keep learning from daily practice to be better managers. They may shift their efforts from community engagement to corporate management. A part-time or full-time community manager then is recruited to engage with the community. At this phase, value of the community shifts from non-buyer core technology developers to buyers who hack the product for new features and new applications. The community demography becomes "Customer innovator". Firms engage with their communities at this phase more for a purpose of generating sales and building up reputation rather than for co-design.

In the phase of business model improvement, open source firms may start to face competition as they have proved that the market is attractive. At this point, they need to further strengthen their core competencies and innovate on their business model. In this phase, firms should have stable financial and manufacturing resources and enough capable internal employees to turn ideas into products in an organized manner. The community is encouraged to develop and share more projects or applications to attract customers in adjacent markets, since more applications means more potential buyers. As the volume of customers increases, community facilitators may help in dealing with the increasing number of the troubleshooting requests, helping to reduce the firm's cost of customer service. The community is now an "active customer community", consisting of application developers, advertisers, active consumers and facilitators.

In the maturation stage, the community still has a big influence on new product ideation, but not so much in technology or strategy. Firms behave more like an established firm. The community becomes a "brand community", whose main contribution is to perform recurrent purchases, product 
ideation and free marketing promotion.

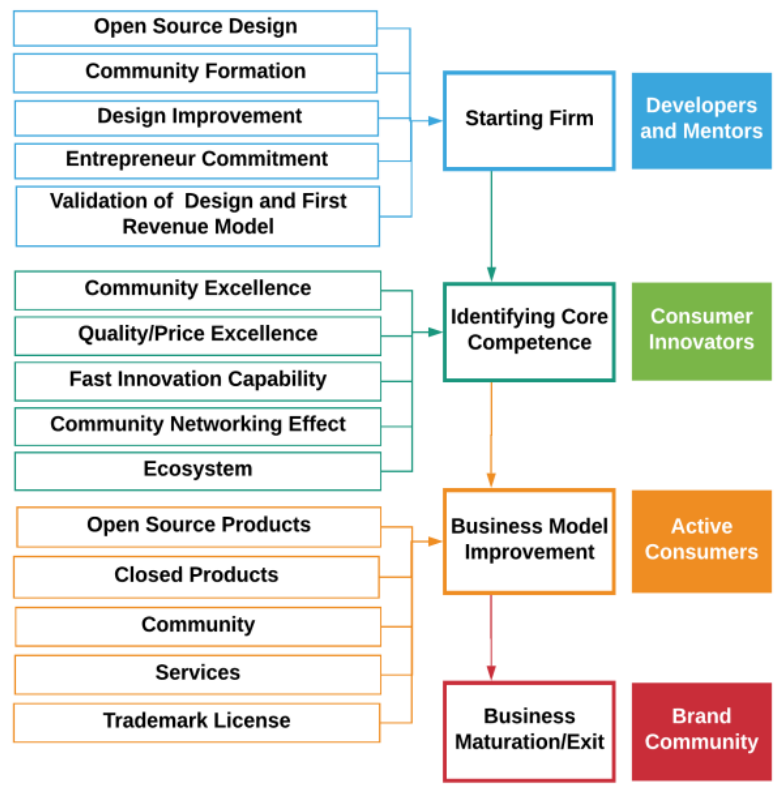

FIGURE 6: IMPACT OF THE COMMUNITY IN EACH PHASE

\section{CONCLUSIONS AND FUTURE RESEARCH}

This paper addresses the question "How do new open source firms build their sustainable business models over time?". We studied 31 open source hardware firms using grounded theory, discovering a 4-phase firm growth path. The path includes Starting the Firm, Building Core Competency, Business Model Improvement and Business Maturation. The community, as the key stakeholder differentiating open source and close source firms, also evolves in its demography and behaviors together with the firm. We explain the community's impact on each phase of the firm's growth and hypothesize that the community's value to the firm changes from product development to marketing and sales.

Future work will involve focusing on each phase and better understanding:

1. What causes entrepreneurs to decide whether or not to pursue the development of an open source product? How does the community influence this decision?

2. How do entrepreneurs identify their core competencies and how do they strengthen them? How does the community influence the identification process?

3. How do entrepreneurs choose and test revenue models? How does the community influence the choice?

4. Quantitative evidence of the evolution of the community's demography, behaviors and value-to-firm in the 4 phases of a firm's growth.

\section{ACKNOWLEDGEMENT}

We would like to acknowledge three anonymous reviewers for the improvement of this paper as well as David Wallace, Maria Yang, Eric von Hippel, Bill Aulet and Scott Stern for inspirations and suggestions.

\section{REFERENCES}

Aulet, W. 2013 Disciplined Entrepreneurship, ISBN:978-1-118-692288 pp. 121-131

Bonvoisin, J. et al., 2017. Current state of practices in open source product development. 21st International Conference On Engineering Design, ICED17, (August), pp.26-28.

Bruijn, E. De, 2010. On the viability of the Open Source Development model for the design of physical objec - Lessons learned from the RepRap project. Master thesis University of Tilburg.

Pacheco-De-Almeida, G. \& Zemsky. P. B.., 2011. Some like it free : innovators ' strategic use of disclosure to slow down competition. Strat. Mgmt. J. DOI: 10.1002/smj

Charmaz, K. (2006). Constructing grounded theory: A practical guide through qualitative analysis. London: Sage Publications.

Chesbrough, H.W. \& Appleyard, M.M., 2007. Open Innovation and Strategy. California Management Review, 50, pp.57-76.

Fjeldsted, A. et al., 2012. Open Source Development of Tangible Products. NordDesign 2012, pp.1-9.

Gupta, P., Guha, S. \& Krishnaswami, S., 2013. Firm growth and its determinants. Journal of Innovation and Entrepreneurship, 2(1).

Haeussler, C., Harhoff, D. \& Mueller, E., 2009. To Be Financed or Not - The Role of Patents for Venture Capital Financing, Working paper: No. 09-003 Center for Europe Economic Research

Osterwalder, A., 2015. The Business Model Generator (2009 edition). ISBN: 978-2-8399-0580-0

Hippel, E., 2007. Horizontal innovation networks - by and for users. Industrial and Corporate Change, pp.1-28.

Howard, T.J. et al., 2012. Open design and crowdsourcing : maturity, methodology and business models. In International Design Conference - Design 2012.

Journal, S.E., Shah, S.K. \& Tripsas, M., 2007. The accidental entrepreneur: the emergent and collective process of user entrepreneurship. Strat. Entrepreneurship J., 1: 123-140.

von Krogh et al., 2012. Carrots and Rainbows: Motivation and Social Practice in Open Source Software Development. MIS Quarterly, 36(2), p.649.

Li, Z.,Seering, W., 2017. Why Open Source ? Exploring the Motivations of Using an Open Design Model for Hardware Development, the ASME International Design Engineering Technical Conferences, Cleveland, $\mathrm{OH}$.

Li, Z.,Seering, W., 2019. Understanding Community Behaviors in Forprofit Open Source Hardware Frims. In International Conference of Engineering and Design, Delft).

Li, Z.,Seering, W., 2019. Value creation and capturing mechanism in open source hardware firms, The International Conference on Engineering Design, Delft .

Moritz, M., Redlich, T. \& Wulfsberg, J.P., 2016. Value Creation in Open-Source Hardware Communities : Case Study of Open Source Ecology. 2016 Portland International Conference on Management of Engineering and Technology

Palacios, M. et al., 2016. Crowdsourcing and organizational forms: Emerging trends and research implications. Journal of Business Research, 69(5), pp.1834-1839.

Pearce, J.M., 2017. Emerging Business Models for Open Source Hardware. Journal of Open Hardware, 1(1), pp.1-14.

Fauchat, É. \& Gruber, M., 2011. Darwinians , communitarians , and missionaries: the role of founder identity in entrepreneurship. Academy of Management Journal 2011, Vol. 54, No. 5, 935-957

Salem, M.A. \& Khatib, J.I., 2004. An Introduction to Open-source Hardware Development. EETimes, pp.1-5.

Yin, R. K. (2003). Case study research: Design and methods. Thousand Oaks, Calif: Sage Publications. 